\title{
Short communication: Evaluation of sampling socks for detection of Mycobacterium avium ssp. paratuberculosis on dairy farms
}

\author{
R. Wolf, ${ }^{*}{ }^{1}$ K. Orsel, $\dagger$ J. De Buck, $\dagger$ U. Kanevets, $\dagger$ and H. W. Barkema $†$ \\ ${ }^{*}$ Amt der Steiermärkischen Landesregierung, 8010 Graz, Austria \\ †Department of Production Animal Health, University of Calgary, Calgary T2N 4N1, Alberta, Canada
}

\begin{abstract}
Mycobacterium avium ssp. paratuberculosis (MAP) causes Johne's disease, a production-limiting disease in cattle. Detection of infected herds is often done using environmental samples (ES) of manure, which are collected in cattle pens and manure storage areas. Disadvantages of the method are that sample accuracy is affected by cattle housing and type of manure storage area. Furthermore, some sampling locations (e.g., manure lagoons) are frequently not readily accessible. However, sampling socks (SO), as used for Salmonella spp. testing in chicken flocks, might be an easy to use and accurate alternative to ES. The objective of the study was to assess accuracy of SO for detection of MAP in dairy herds. At each of 102 participating herds, $6 \mathrm{ES}$ and $2 \mathrm{SO}$ were collected. In total, 45 herds had only negative samples in both methods and 29 herds had $\geq 1$ positive ES and $\geq 1$ positive $S O$. Furthermore, 27 herds with $\geq 1$ positive ES had no positive SO, and 1 herd with no positive ES had 1 positive SO. Bayesian simulation with informative priors on sensitivity of ES and MAP herd prevalence provided a posterior sensitivity for SO of $43.5 \%$ (95\% probability interval = $33-58$ ), and $78.5 \%$ (95\% probability interval $=62-93$ ) for ES. Although SO were easy to use, accuracy was lower than for ES. Therefore, with improvements in the sampling protocol (e.g., more SO per farm and more frequent herd visits), as well as improvements in the laboratory protocol, perhaps $\mathrm{SO}$ would be a useful alternative for ES.
\end{abstract}

Key words: paratuberculosis, Johne's disease, environmental samples, sock samples

\section{Short Communication}

Mycobacterium avium ssp. paratuberculosis (MAP) causes Johne's disease (JD), a production-limiting dis-

Received August 19, 2015.

Accepted December 20, 2015.

${ }^{1}$ Corresponding author: robert.wolf@stmk.gv.at ease in cattle (Fecteau and Whitlock, 2010). That, as well as the potentially zoonotic nature of the pathogen, motivate control efforts (Barkema et al., 2010). Environmental sampling (ES) of manure is frequently used for detection of MAP-infected herds. For that method, manure is collected from pens and manure storage areas and analyzed for MAP using culture or PCR (Wolf et al., 2014a). However, accuracy depends on the situation on the farm, because samples from manure lagoons are more accurate than samples from manure piles, and samples from alleys are more accurate than samples from bedding packs (Wolf et al., 2015a). Furthermore, sample collection from manure storage areas can be difficult because they are not always readily accessible. Sock samples (SO), as used for detection of Salmonella spp. in poultry (Skov et al., 1999), might be an accurate and more convenient alternative to conventional environmental samples; in tests in a few high-prevalence herds, SO detected MAP (Eisenberg et al., 2013). Because samples are collected with every step of the sampler, SO might be more accurate than the standard ES. The objective of the current study was to assess accuracy of SO for detection of MAP in dairy herds.

Participating herds were part of the Alberta Johne's Disease Initiative (AJDI), a MAP-control program with $62 \%$ of the 594 Alberta dairy farmers participating (Wolf et al., 2014b). For the present study, veterinarians of the 4 veterinary clinics with the highest number of participants in the AJDI were asked to recruit all AJDI herds where visits were scheduled between June and October 2013. Additionally, farms visited by University of Calgary personnel for a study on MAP shedding in calves (Wolf et al., 2015b), as well as farms that participated in a MAP survey study (Ritter et al., 2014), were also included. Sample collectors were trained for ES collection during a half-day workshop and were instructed for SO collection with a one-page instruction sheet. To facilitate laboratory procedures, herds were only visited between Monday and Wednesday; 6 ES were collected following a standardized protocol (Wolf et al., 2014a). In short, samples were collected from 
(1) lactating cow pens, (2) manure storage areas, and (3) dry, sick, or calving pens. Although 2 samples were collected at each location, locations were replaced if they were not accessible or did not qualify $(<2$ cows in a pen). Each sample contained at least 4 subsamples that were mixed in a plastic bag. Additionally, $2 \mathrm{SO}$ were collected on each participating farm. The SO were assembled using single-use cover boots and a commercially available dust swipe $(\sim 12 \times 12 \mathrm{~cm})$ taped on the bottom of the boot. Sample collectors walked once up and down the lactating cow alley, wearing the cover boots on top of their normal footwear. After sample collection, dust swipes were removed from the cover boot and stored in plastic bags.

All samples were sent (express mail) to the University of Calgary within $24 \mathrm{~h}$ after collection. Upon arrival, they were stored at $4^{\circ} \mathrm{C}$ and processed within $8 \mathrm{~d}$ after sample collection. Samples were processed using a TREK ESP culture protocol (Mortier et al., 2014). The only difference between the ES and SO protocol was the first step of the decontamination protocol, producing a $5 \%$ manure in water solution. For ES, $2 \mathrm{~g}$ of sample material was added to water, whereas for SO the difference between the weight of an unused $\mathrm{SO}$ and the weight of the used $\mathrm{SO}$ was determined and water was added proportionally. Thereafter, ES and SO went through $3 \mathrm{~d}$ of decontamination and $42 \mathrm{~d}$ of bacterial culture, all ES and SO culture products were analyzed using conventional IS900 PCR (Vary et al., 1990). The PCR result was used as a definition of whether a sample was positive or negative for MAP.

For a farm-level test comparison, a farm was defined as ES-positive if at least 1 of 6 ES was MAP-positive, or as SO-positive if at least 1 of $2 \mathrm{SO}$ were MAP-positive. Sensitivity of SO to detect a MAP-positive farm was determined using a 2-dependent test and 1-population Bayesian analysis model in WinBugs (Branscum et al., 2005; Lunn et al., 2000; Appendix). Informative priors were used for herd prevalence of MAP in Alberta (Mode: $68 \%, 80 \%$ certain $>56 \%$ ) and sensitivity of 6 ES (Mode: 68\%, 90\%, certain that $<82 \%$ ) in accordance with a previous study (Wolf et al., 2014a). It was assumed that both test methods had 1 out of 1,000 false-positive farms.

For sensitivity analysis, independence between tests was assumed (model 2), uninformative priors were used in separate models (models 3 and 4), and a 2-population approach was used (model 5), dividing herds into those where the farmer had previously observed cows with signs of clinical JD (high-risk population) and farms without observation of cows with signs consistent with clinical JD (low-risk population). Additionally, these populations were analyzed in 2 separate 1-population models (models 6 and 7 ).
A total of 102 herds participated in the study, with a mean herd size of 145 cows $(\mathrm{SD}=80$; minimum $=34$; maximum $=474)$. Of those, 45 herds $(44 \%)$ only had negative samples in both methods, whereas 29 herds $(28 \%)$ tested positive in both sampling strategies, confirming that $\mathrm{SO}$ can be used to detect MAP-infected herds. A total of 27 (27\%) ES-positive herds were not detected with SO, whereas 1 SO-positive herd (1\%) had no positive ES (Figure 1).

Posterior sensitivity for SO was 44\% [95\% probability interval $(\mathbf{P I})=33-58 \%$, whereas it was $79 \%(95 \%$ $\mathrm{PI}=62-93 \%$ ) for ES. An obvious reason for the SO to have lower sensitivity than the ES is that a sample set consisted of $6 \mathrm{ES}$, but only $2 \mathrm{SO}$ were included per farm. More samples per farm would likely increase sensitivity (Dohoo et al., 2003). In the present study, only $2 \mathrm{SO}$ were collected per farm, as this number of samples would make this method more practical and cost-effective. Furthermore, we tried to avoid major increases in workloads for AJDI veterinarians by asking them to collect only limited numbers of additional samples; however, accuracy of $\mathrm{SO}$ protocols with $>2$ samples should be evaluated in a future study. An alternative sampling strategy for replacing ES completely would be to use SO for lactating cow pens, but to still use ES to additionally sample manure storage areas and dry/ sick and calving pens. However, this alternative could still result in lower accuracy, because 2 SO detected a smaller number of infected herds ( 27 herds) than 2 lactating cow pen ES (40 herds; Table 1). This was surprising, because it was assumed that false-negative ES were mainly caused by ES not containing manure of MAP-shedding cows. Therefore, an increase in the number of subsamples per sample through usage of $\mathrm{SO}$ was expected to increase sensitivity. In that regard, individual SO should have been more accurate than individual ES, because subsamples were theoretically collected with every step. However, a likely reason for the low accuracy was that the absorbent material on the $\mathrm{SO}$ was saturated after the first steps in the pen because dairy cow manure is very liquid. A possible solution would have been to use a thicker material (e.g., a sponge) that would have absorbed and released manure with every step, collecting material throughout the entire alley. An explanation for lower accuracy was that collected manure may not have readily dispersed in water during laboratory processing, resulting in less manure included during bacterial culture of SO than ES. However, that could be overcome with more intense mechanical treatment of the absorbent material. Another concern using SO might be that the socks would not collect enough manure for MAP culture. However, the net amount of collected manure in SO always exceeded $2 \mathrm{~g}$, which is the amount used for culturing ES. 


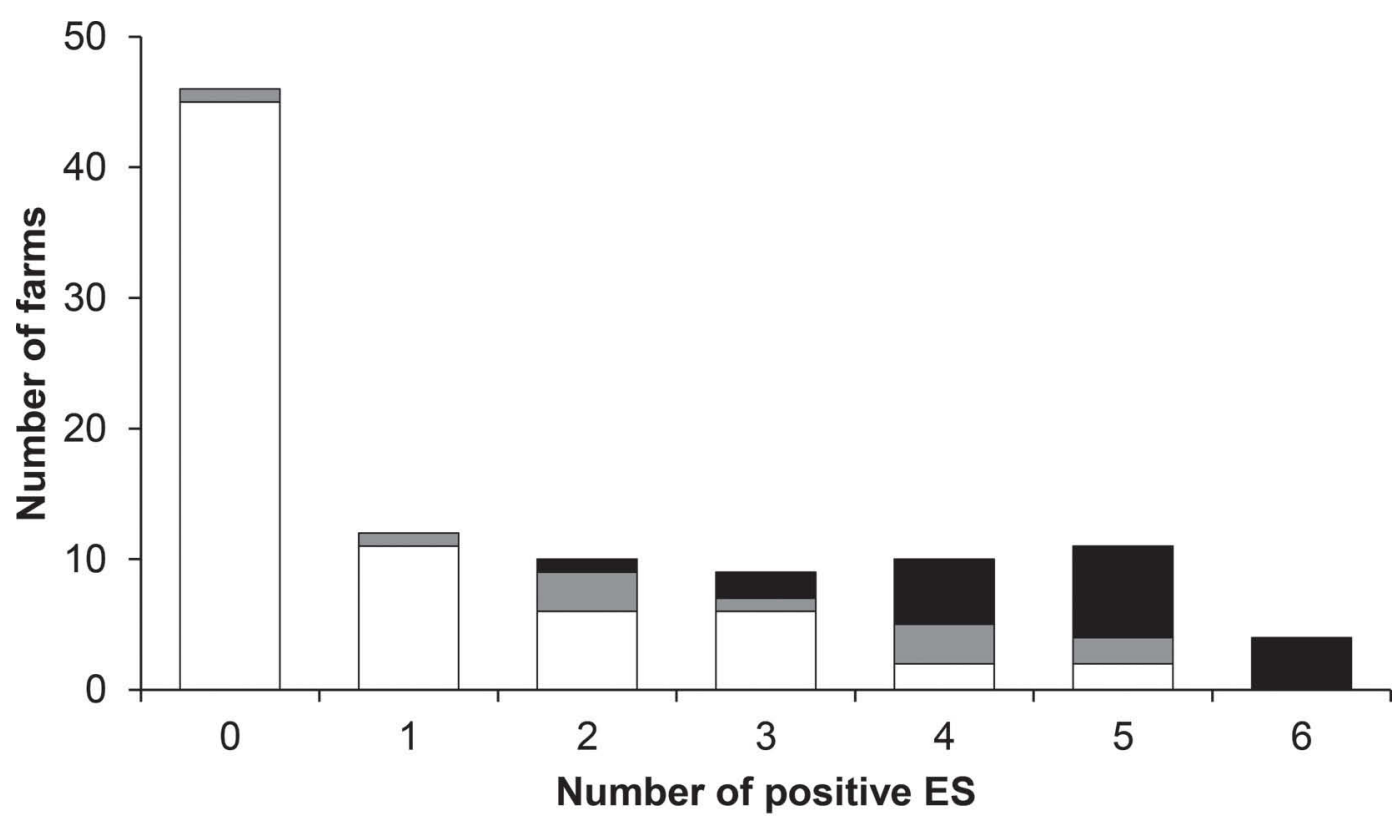

$\square 0$ positive SO $\square 1$ positive SO $\quad \mathbf{2}$ positive SO

Figure 1. Number of Mycobacterium avium ssp. paratuberculosis-positive environmental samples (ES) and number of positive sock samples (SO) collected on 102 dairy farms.

The prevalence of MAP-infected herds was $69 \%$ (95\% $\mathrm{PI}=54-84 \%$ ), similar to MAP prevalence in all AJDI herds (Wolf et al., 2014a), suggesting that participating herds were representative of all herds in the AJDI. Sensitivity of ES was higher than in a previous publication, perhaps due to optimization of the ES laboratory protocol that occurred between the 2 studies (Wolf et al., 2014a). Specificity for both SO and ES was $>99.0 \%$, due to high prior specificity, supported by the fact that both tests are direct detection methods.

The use of uninformative priors affected posterior estimates to a small extent (models 3 and 4 in Table 2). Therefore, the analysis relied on prior information on the accuracy of ES and herd prevalence due to the high number of estimated parameters and few degrees of freedom (Branscum et al., 2005). Both parameters, sensitivity of ES and herd prevalence, were estimated for the same population and the same laboratory protocol in a recent study (Wolf et al., 2014a), which provided confidence regarding accuracy of estimates.

Separate analyses of high- and low-risk populations (models 6 and 7 in Table 2) resulted in SO sensitivity posteriors of 53 and $30 \%$, respectively $(95 \% \mathrm{PI}=37-70$ and $13-52 \%$, respectively). The same trend was observed for ES, which suggests that the accuracy of both methods depended on within-herd MAP prevalence, which was likely higher in herds with a history of clinical JD (high-risk population; model 7) than in herds

Table 1. Comparison of Mycobacterium avium ssp. paratuberculosis culture results (pos., positive) between 2 sock samples (SO) and 2 environmental samples collected from lactating cow pens, manure storage, and dry, sick, and calving pens ${ }^{1}$

\begin{tabular}{|c|c|c|c|c|c|c|c|c|c|}
\hline \multirow[b]{2}{*}{ Item } & \multicolumn{3}{|c|}{$\begin{array}{l}\text { Lactating cow pens } \\
\qquad(\mathrm{n}=95)\end{array}$} & \multicolumn{3}{|c|}{$\begin{array}{l}\text { Manure storage } \\
\quad(\mathrm{n}=63)\end{array}$} & \multicolumn{3}{|c|}{$\begin{array}{l}\text { Dry, sick, and calving pens } \\
\qquad(\mathrm{n}=51)\end{array}$} \\
\hline & 0 & 1 & 2 & 0 & 1 & 2 & 0 & 1 & 2 \\
\hline 0 pos. SO & 54 & 11 & 3 & 32 & 4 & 7 & 30 & 9 & 0 \\
\hline 1 pos. SO & 1 & 4 & 4 & 2 & 2 & 3 & 4 & 0 & 1 \\
\hline 2 pos. SO & 0 & 3 & 15 & 4 & 2 & 7 & 1 & 4 & 2 \\
\hline
\end{tabular}

${ }^{1}$ Herds were included if at least 2 samples were collected from the specified location. If $>2$ samples were submitted from the same location, 2 samples were chosen randomly. 
SHORT COMMUNICATION: DETECTING PARATUBERCULOSIS

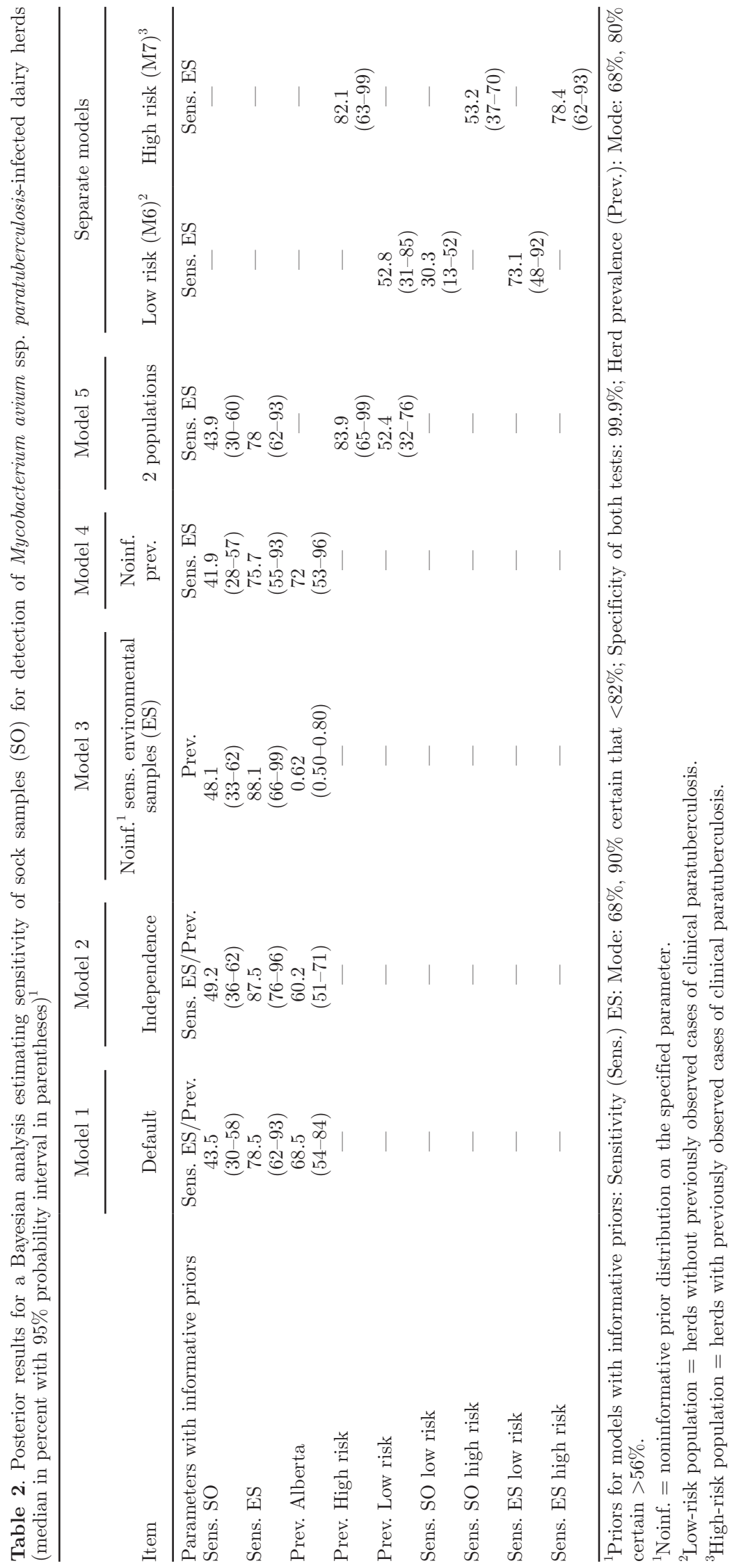


without observed cases of clinical JD (low-risk population, model 6). This confirmed other studies reporting higher sensitivity of ES and SO in high-prevalence herds (Tavornpanich et al., 2008; Lavers et al., 2013; Donat et al., 2016). This within-herd prevalence dependent sensitivity should be considered when generalizing results to other populations, because ES and SO are expected to be more accurate in populations with more high-prevalence herds.

In conclusion, $\mathrm{SO}$ were less accurate than ES for detection of MAP-infected dairy herds. However, SO accuracy might be increased by collection of more SO per farm and optimization of SO design and the laboratory protocol. Ultimately, these improvements could result in a cheap and easy, yet accurate method to detect MAP-infected herds.

\section{ACKNOWLEDGMENTS}

The authors thank farmers and veterinarians for participating. This study was funded by Alberta Milk and the Canadian Agricultural Adaptation Program (CAAP; Ottawa, ON, Canada). Thank you to John Kastelic (University of Calgary) for editing the manuscript.

\section{REFERENCES}

Barkema, H. W., S. Hendrick, J. De Buck, G. G. Kaplan, and K. Rioux. 2010. Crohn's disease in humans and Johne's disease in cattle - Linked diseases? Pages 197-213 in Zoonotic Pathogens in the Food Chain. D. Krause and S. Hendrick, ed. CAB International, Wallingford, UK.

Branscum, A. J., I. A. Gardner, and W. O. Johnson. 2005. Estimation of diagnostic-test sensitivity and specificity through Bayesian modeling. Prev. Vet. Med. 68:145-163.

Dohoo, I. R., W. Martin, and H. Stryhn. 2003. Sampling. Pages 27-52 in Veterinary Epidemiologic Research. M. S. McPike, ed. Atlantic Veterinary College, Charlottetown, PEI, Canada.

Donat, K., N. Hahn, T. Eisenberg, K. Schlez, H. Koehler, W. Wolter, M. Rohde, R. Puetzschel, U. Roesler, K. Failing, and P. M. Zschoeck. 2016. Within-herd prevalence thresholds for the detection of Mycobacterium avium subspecies paratuberculosis-positive dairy herds using boot swabs and liquid manure samples. Epidemiol. Infect. 144:413-424.
Eisenberg, T., W. Wilfried, M. Rohde, K. Schlez, and M. Zschoeck. 2013. Boot swabs to collect environmental samples from common locations in dairy herds for Mycobacterium avium sssp. paratuberculosis (MAP) detection. J. Dairy Res. 80:485-489.

Fecteau, M. E., and R. H. Whitlock. 2010. Paratuberculosis in cattle. Pages 144-156 in Paratuberculosis - Organism, Disease and Control. M. A. Behr and D. M. Collins, ed. CABI, Wallingford, UK.

Lavers, C. J., S. L. B. McKenna, I. R. Dohoo, H. W. Barkema, and G. P. Keefe. 2013. Evaluation of environmental fecal culture for $M y-$ cobacterium avium subspecies paratuberculosis detection in dairy herds and association with apparent within-herd prevalence. Can. Vet. J. 54:1053-1060.

Lunn, D. J., A. Thomas, N. Best, and D. Spiegelhalter. 2000. WinBUGS-A Bayesian modelling framework: Concepts, structure, and extensibility. Stat. Comput. 10:325-337.

Mortier, R. A. R., H. W. Barkema, K. Orsel, R. Wolf, and J. De Buck. 2014. Shedding patterns of dairy calves experimentally infected with Mycobacterium avium subspecies paratuberculosis. Vet. Res. 45:71-80.

Ritter, C., R. Wolf, B. S. Mason, J. Flaig, M. Slomp, C. Pickel, and H. W. Barkema. 2014. Extension communication preferences of Alberta dairy producers. Page 113 in Proc. 12th Intern. Colloq. on Paratuberculosis (ICP), Parma, Italy. International Association for Paratuberculosis, Parma, Italy.

Skov, M. N., B. Carstensen, N. Tornøe, and M. Madsen. 1999. Evaluation of sampling methods for the detection of Salmonella in broiler flocks. J. Appl. Microbiol. 86:695-700.

Tavornpanich, S., C. A. Muñoz-Zanzi, S. J. Wells, E. A. Raizman, T. E. Carpenter, W. O. Johnson, and I. A. Gardner. 2008. Simulation model for evaluation of testing strategies for detection of paratuberculosis in Midwestern US dairy herds. Prev. Vet. Med. 83:65-82.

Vary, P. H., P. R. Andersen, E. Green, J. Hermon-Taylor, and J. J. McFadden. 1990. Use of highly specific DNA probes and the polymerase chain reaction to detect Mycobacterium paratuberculosis in Johne's disease. J. Clin. Microbiol. 28:933-937.

Wolf, R., H. W. Barkema, J. De Buck, and K. Orsel. 2015a. Sampling location, herd size and season influence Mycobacterium avium subspecies paratuberculosis environmental culture results. J. Dairy Sci. 98:275-287.

Wolf, R., H. W. Barkema, J. De Buck, M. Slomp, J. Flaig, D Haupstein, C. Pickel, and K. Orsel. 2014a. High herd-level prevalence of Mycobacterium avium subspecies paratuberculosis in Western Canadian dairy farms, based on environmental sampling. J. Dairy Sci. 97:6250-6259.

Wolf, R., F. Clement, H. W. Barkema, and K. Orsel. 2014b. Economic evaluation of participation in a voluntary Johne's disease prevention and control program from a farmer's perspective - The Alberta Johne's Disease Initiative. J. Dairy Sci. 97:2822-2834.

Wolf, R., K. Orsel, J. De Buck, and H. W. Barkema. 2015b. Calves shedding Mycobacterium avium subspecies paratuberculosis are common on infected dairy farms. Vet. Res. 46:71-79. 


\section{APPENDIX}

WinBugs code for 2-dependent test and 1-population Bayesian analysis model used to estimate the sensitivity of sock samples (SO) compared with environmental samples (ES) for the detection of Mycobacterium avium ssp. paratuberculosis-infected dairy herds.

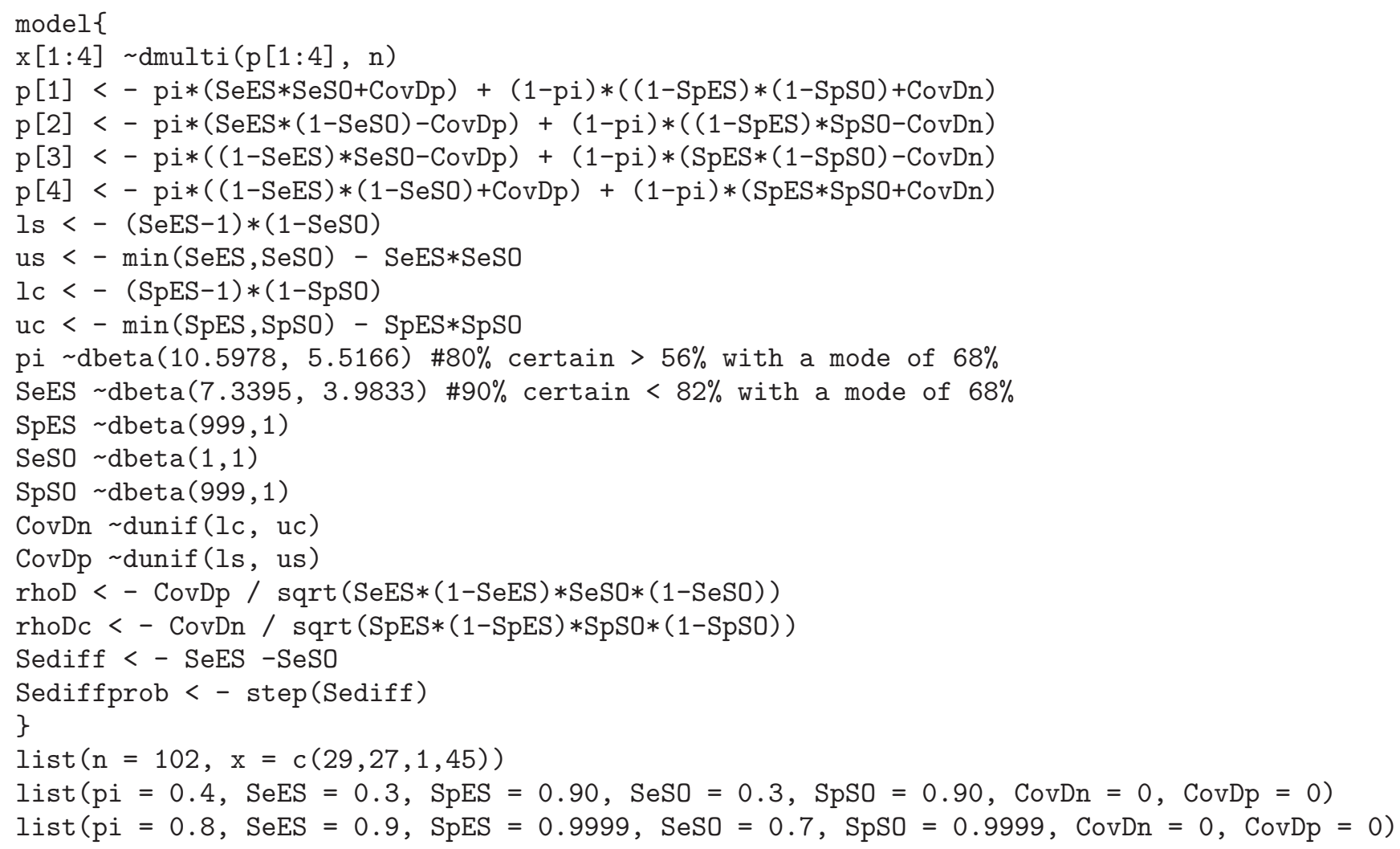

